\title{
Mandibular advancement oral appliance therapy for obstructive sleep apnoea: effect on awake calibre of the velopharynx
}

\author{
C F Ryan, L L Love, D Peat, J A Fleetham, A A Lowe
}

\begin{abstract}
Background-The mechanisms of action of oral appliance therapy in obstructive sleep apnoea are poorly understood. Videoendoscopy of the upper airway was used during wakefulness to examine whether the changes in pharyngeal dimensions produced by a mandibular advancement oral appliance are related to the improvement in the severity of obstructive sleep apnoea.
\end{abstract}

Methods-Fifteen patients with mild to moderate obstructive sleep apnoea (median (range) apnoea index (AI) 4(0-38)/h, apnoea-hypopnoea index (AHI) 28(945)/h) underwent overnight polysomnography and imaging of the upper airway before and after insertion of the oral appliance. Images were obtained in the hypopharynx, oropharynx, and velopharynx at end tidal expiration during quiet nasal breathing in the supine position. The cross sectional area and diameters of the upper airway were measured using image processing software with an intraluminal catheter as a linear calibration. Results-AI decreased to a median (range) value of $0 \quad(0-6) / \mathrm{h} \quad(\mathrm{p}<0.01)$ and AHI to $8(1-28) / h \quad(p<0.001)$ following insertion of the oral appliance. The median $(95 \%$ confidence interval) cross sectional area of the upper airway increased by $18 \%$ ( 3 to 35$)(p<0.02)$ in the hypopharynx and by $25 \%(11$ to 69$)(\mathrm{p}<0.005)$ in the velopharynx, but not significantly in the oropharynx. Although in general the shape of the pharynx did not change following insertion of the oral appliance, the lateral diameter of the velopharynx increased to a greater extent than the anteroposterior diameter. Following insertion of the oral appliance the reduction in AHI was related to the increase in cross sectional area of the velopharynx ( $p=$ 0.01).

Conclusions-A mandibular advancement oral appliance increases the cross sectional area of the upper airway during wakefulness, particularly in the velopharynx. Assuming this effect on upper airway calibre is not eliminated by sleep, mandibular advancement oral appliances may reduce the severity of obstructive sleep apnoea by maintaining patency of the velopharynx, particularly in its lateral dimension.

(Thorax 1999;54:972-977)
Keywords: obstructive sleep apnoea; mandibular advancement oral appliance

Oral appliances are an interesting new approach to the treatment of sleep disordered breathing. ${ }^{1}$ They are indicated for the treatment of simple snoring and mild obstructive sleep apnoea, and for moderate to severe obstructive sleep apnoea when nasal continuous positive airway pressure (CPAP) therapy is not tolerated and upper airway surgery is not indicated. ${ }^{2}$ Most oral appliances are designed to maintain the mandible and/or tongue in a protruded posture during sleep, thereby preventing upper airway occlusion. ${ }^{3-7}$ The precise mode and site of action of oral appliances remains unclear, however. Proposed mechanisms of action include increased upper airway calibre, decreased upper airway compliance, or activation of upper airway dilator muscles. Oral appliances were believed to exert their effects predominantly in the oropharynx and hypopharynx but some studies have suggested an effect on the retropalatal airway also. ${ }^{58}$

Several studies have demonstrated an increase in the anteroposterior diameter of the upper airway following insertion of an oral appliance. ${ }^{5-9}$ Whether these changes were associated with an increase in the cross sectional area (CSA) of the upper airway is unclear. In a previous report of a single patient ${ }^{10}$ we have shown, using cephalometry and three dimensional computed tomographic reconstructions, that a mandibular advancement oral appliance increased the posterior airway space from $9.4 \mathrm{~mm}$ to $10.2 \mathrm{~mm}$, and the minimum CSA of the upper airway from $41.6 \mathrm{~mm}^{2}$ to $92.3 \mathrm{~mm}^{2}$. We have recently shown that voluntary mandibular and tongue protrusion increases the CSA of the upper airway and alters its shape in patients with obstructive sleep apnoea and non-apnoeic control subjects. ${ }^{11}$

Previous studies have documented the efficacy of mandibular advancement oral appliances in the treatment of obstructive sleep apnoea. ${ }^{12-14}$ The purpose of the present study was to measure the regional effects of a mandibular advancement oral appliance on awake upper airway calibre using videoendoscopy. We hypothesised that an adjustable mandibular advancement oral appliance would increase the CSA of the upper airway, and that the increase in airway calibre would be related to the efficacy of the oral appliance in reducing the severity of obstructive sleep apnoea. 


\section{Methods}

SUBJECTS

Fifteen patients with symptomatic mild to moderate obstructive sleep apnoea were recruited through the Sleep Disorders Program at Vancouver Hospital and Health Sciences Centre following diagnostic overnight polysomnography. Patients with severe obstructive sleep apnoea were strongly encouraged to pursue nasal CPAP therapy because of its established safety and efficacy. After a full discussion of the various therapeutic options, consecutive patients who elected to be treated with an oral appliance were considered for inclusion in the study. Prior to recruitment the patients underwent a detailed clinical orthodontic and craniofacial evaluation to determine their suitability for oral appliance therapy. Patients with inadequate dentition, gingivitis, temporomandibular joint dysfunction, or severe nasal obstruction were excluded. Each patient gave written informed consent and the study protocol was approved by the ethical review committee of our institution.

\section{STUDY DESIGN}

Each patient had an overnight polysomnogram performed prior to enrolment in the study. Polysomnography was repeated with the oral appliance in place at least two months after the patient had been fitted with the oral appliance and once it had been adjusted to the maximum degree of protrusion consistent with the patient's comfort and ability to wear it on a nightly basis. On the morning after the second polysomnogram each patientunderwentavideoendoscopic examination of the upper airway, first without the oral appliance and then with the oral appliance in place.

\section{POLYSOMNOGRAPHY}

Sleep and its various stages were documented by standard electroencephalographic (EEG), electro-oculographic (EOG), and electromyographic (EMG) criteria. $^{15}$ EEG was recorded with electrodes applied at $\mathrm{C}_{3}-\mathrm{A}_{2}$ and $\mathrm{C}_{4}-\mathrm{A}_{1}$ (according to the International 10-20 system) and EMG activity was recorded from the submental muscles and anterior tibialis muscles. Apnoeas were documented by an infrared $\mathrm{CO}_{2}$ analyser (Model 1260; Novamatrix Medical Systems Inc, Wallingford, Connecticut, USA) which records from both the nose and mouth. A single electrocardiographic (ECG) lead (modified $\mathrm{V}_{2}$ ) was monitored to detect cardiac arrhythmias. Arterial oxygen saturation $\left(\mathrm{SaO}_{2}\right)$ was monitored continuously with a pulse oximeter (Model N-100; Nellcor Inc, Hayward, California, USA) attached to the index finger. Chest wall movement was monitored by a respiratory inductive plethysmograph (Respitrace; Respitrace Corporation, Ardsley, New York, USA). The data were recorded on a 15 channel polygraph (Model 78; Grass Instruments Co, Quincy, Massachusetts, USA) and fed into the CNS Sleep Lab System (Model 200; CNS Inc, Chanhassen, Minnesota, USA). The complete record was manually scored for sleep stage and apnoea type and duration. Severity of sleep apnoea was assessed in terms
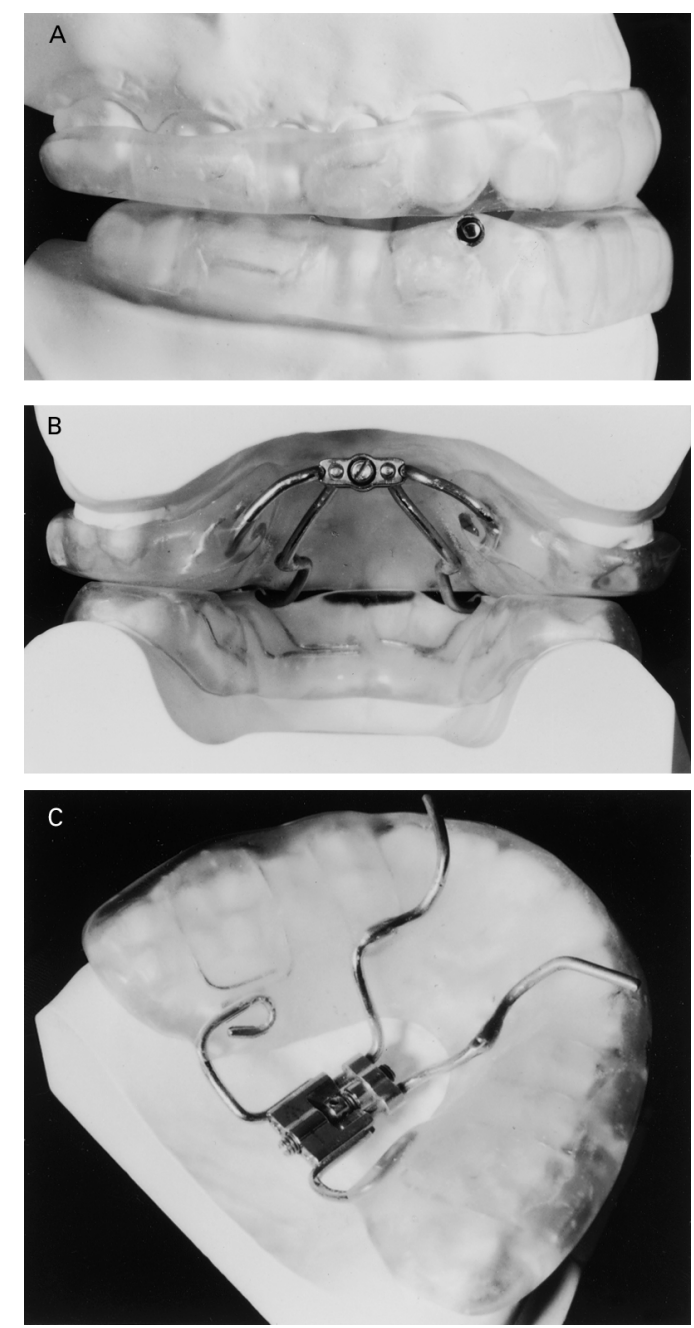

Figure 1 (A) Lateral, (B) posterior and (C) palatal views of the Klearway ${ }^{T M}$ mandibular advancement oral appliance. The adjustable screw mechanism, which is recessed in the palatal arch, permits $11 \mathrm{~mm}$ mandibular protrusion in 44 increments of $0.25 \mathrm{~mm}$ and also allows vertical and horizontal jaw movement.

of number of apnoeas and number of apnoeas + hypopnoeas/total sleep time and expressed as the apnoea index (AI) and the apnoea + hypopnoea index (AHI), respectively.

ORAL APPLIANCE

Each patient was fitted with a Klearway ${ }^{\mathrm{TM}}$ oral appliance (Space Maintainers Laboratories Canada Ltd, Vancouver, BC; fig 1). The Klearway $^{\mathrm{TM}}$ is a new adjustable anterior mandibular advancement oral appliance developed by one of the authors (AAL). It is a custom built oral appliance fashioned from a thermoactive acrylic resin that becomes more compliant with heating. This design facilitates easier insertion and increases retention during sleep. The Klearway ${ }^{\mathrm{TM}}$ anchors on the maxillary and mandibular dental arches and provides complete occlusal coverage. The maxillary and mandibular components are joined by an adjustable screw mechanism that provides for a gradual protrusion of the mandibular component in increments of $0.25 \mathrm{~mm}$ over a total range of $11 \mathrm{~mm}$. The design of the screw mechanism allows for $1-3 \mathrm{~mm}$ of lateral jaw movement and 1-5 mm of vertical jaw movement, thereby reducing the risk of temporomandibular joint and jaw muscle discomfort 
Table 1 Anthropometric and polysomnographic data before and after oral appliance emplacement

\begin{tabular}{lllll}
\hline & Before & After & Differencet & p value $\neq$ \\
\hline M:F & $12: 3$ & & & \\
Age (years) & $52(25-70)$ & & & \\
Neck circumference $(\mathrm{cm})$ & $43(34-48)$ & & & \\
Body mass index $\left(\mathrm{kg} / \mathrm{m}^{2}\right)$ & $32(23-65)$ & $34(23-65)$ & $0(-0.7$ to 0.5$)$ & NS \\
AI (/h TST) & $4(0-38)$ & $0(0-6)$ & $4(2$ to 13$)$ & $<0.01$ \\
AHI (/h TST) & $28(9-45)$ & $8(1-28)$ & $17(11$ to 25$)$ & $<0.001$ \\
\hline
\end{tabular}

$\mathrm{AI}=$ apnoea index $; \mathrm{AHI}=$ apnoea + hypopnoea index; $\mathrm{TST}=$ total sleep time.

*Median (range).

†Median (95\% confidence intervals).

‡Wilcoxon signed ranks test. analysis. Specific anatomical levels were measured in sequence beginning at the hypopharynx (just distal to the tip of the epiglottis), followed by the oropharynx (between the uvula and the epiglottis), and finally the velopharynx (just proximal to the free margin of the soft palate). Images were first obtained without the oral appliance and then following its insertion.

IMAGE ANALYSIS

The analysis of the videoendoscopic images was performed by one investigator (LLL), blinded to the polysomnographic data, and using the technique which we have previously described. ${ }^{11}$ Airway CSA, anteroposterior and lateral diameters were measured from the stored images using a high resolution frame grabber (Model DT3851; Data Translation, Marlboro, Massachusetts, USA) and image processing software (Global Lab Image: Data Translation). Signals from the time code generator and oesophageal pressure transducer were stored on-line in a computer (80486; ANO Automation, Vancouver, BC) enabling selection of images based on simultaneous pressure measurements. Measurements were made at end tidal expiration as determined by the oesophageal pressure transducer. The airway lumen was traced freehand to generate a region of interest that was quantified based on the number of enclosed pixels. The border between the airway and soft tissues was determined visually. To ensure consistency of location of the traced images before and after oral appliance emplacement, unique mucosal landmarks on the posterior pharyngeal wall in each patient were used. All measurements of CSA were made using the diameter of the intraluminal catheter as a linear calibration. Measurements carried out in duplicate for 15 separate determinations of CSA, five each in the hypopharynx, oropharynx and velopharynx, yielded a coefficient of variation of 3.8\%. Errors of less than $5 \%$ were obtained when measurements using this tracing technique were performed on images of tubes of known CSA ranging between 1 and $4 \mathrm{~cm}^{2}$. Only images which encompassed the entire upper airway lumen were included in the analysis, resulting in the loss of data in the hypopharynx and oropharynx in one patient. Upper airway

Table 2 Upper airway dimensions before and after insertion of the oral appliance

\begin{tabular}{|c|c|c|c|c|c|}
\hline & Before * & After* & Changet & $\%$ changet & $p$ value $\neq$ \\
\hline \multicolumn{6}{|c|}{ Cross sectional area $\left(\mathrm{mm}^{2}\right)$} \\
\hline Hypopharynx & $67(12-237)$ & $64(34-251)$ & $126(109$ to 181$)$ & 18 (3 to 35$)$ & $<0.02$ \\
\hline Oropharynx & $103(39-235)$ & $115(40-297)$ & $12(-5$ to 45$)$ & $11(-1$ to 43$)$ & NS \\
\hline Velopharynx & $96(43-281)$ & $126(57-283)$ & $24(10$ to 44$)$ & $25(11$ to 69$)$ & $<0.005$ \\
\hline \multicolumn{6}{|c|}{ Anteroposterior diameter (mm) } \\
\hline Hypopharynx & $9.4(3.0-12.5)$ & $11.0(4.2-14.4)$ & $1.1(-0.2$ to 2.4$)$ & $12(-1$ to 36$)$ & NS \\
\hline Oropharynx & $9.6(4.2-15.1)$ & $10.2(7.3-16.4)$ & $1.1(-0.16$ to 2.28$)$ & $8(-3$ to 39$)$ & NS \\
\hline Velopharynx & $7.8(4.7-19.9)$ & $9.6(5.8-21.4)$ & $1.4(-0.7$ to 3.4$)$ & $26(-1$ to 43$)$ & NS \\
\hline \multicolumn{6}{|c|}{ Lateral diameter (mm) } \\
\hline Hypopharynx & $11.6(5.3-24.3)$ & $13.2(7.6-23.7)$ & $1.2(-0.3$ to 2.3$)$ & $7(-6$ to 31$)$ & NS \\
\hline Oropharynx & $17.4(11.9-22.2)$ & $18.2(11.5-27.0)$ & $1.0(-1.6$ to 3.4$)$ & $6(-8$ to 19$)$ & NS \\
\hline Velopharynx & $14.5(7.4-28.1)$ & $17.0(9.9-33.9)$ & $2.5(1.0$ to 4.2$)$ & $14(8$ to 33$)$ & $<0.005$ \\
\hline \multicolumn{6}{|c|}{ Anteroposterior/lateral diameter } \\
\hline Hypopharynx & $0.74(0.24-1.65)$ & $0.60(0.25-1.34)$ & $0.0(-0.2$ to 0.1$)$ & & NS \\
\hline Oropharynx & $0.53(0.25-0.86)$ & $0.53(0.39-0.78)$ & $0.0(-0.05$ to 0.08$)$ & & NS \\
\hline Velopharynx & $0.49(0.24-1.87)$ & $0.51(0.32-1.44)$ & $0.04(-0.2$ to 0.10$)$ & & NS \\
\hline
\end{tabular}

*Median (range)

† Median ( $95 \%$ confidence intervals).

$\ddagger$ Wilcoxon signed ranks test. 


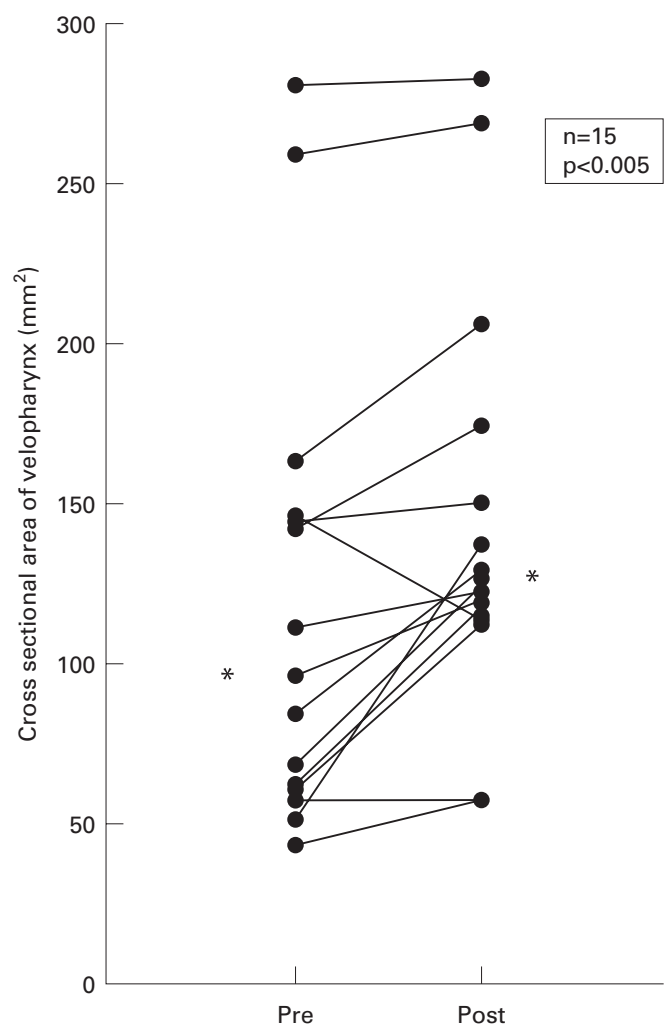

Figure 2 Increase in cross sectional area of the velopharynx after insertion of the Klearway ${ }^{T M}$ oral appliance. ${ }^{\star}$ Median values.

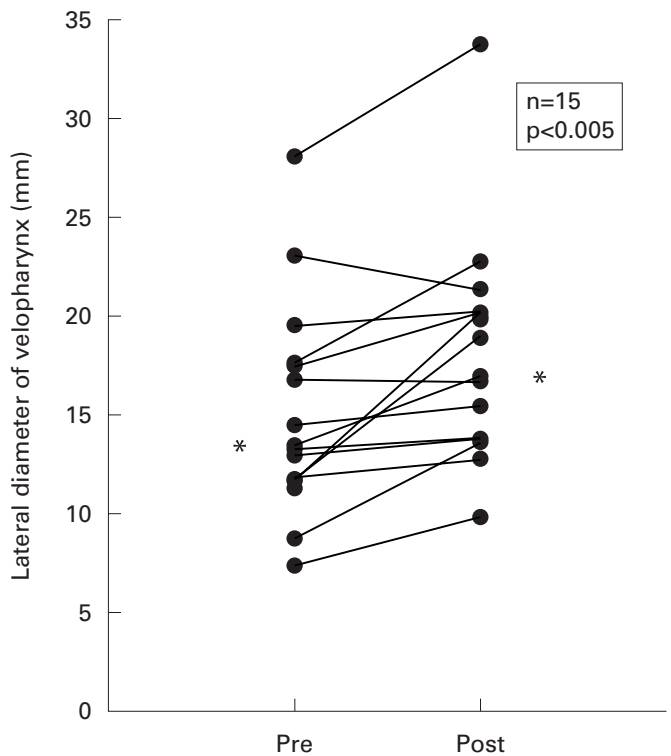

Figure 3 Increase in lateral diameter of the velopharynx after insertion of the Klearway ${ }^{T M}$ oral appliance. ${ }^{\star}$ Median values.

shape was expressed as the ratio of the anteroposterior to lateral diameters such that values close to 1.0 indicated a more circular shape.

STATISTICAL ANALYSIS

Changes in polysomnographic and videoendoscopic variables before and after insertion of the oral appliance were compared using a two tailed Wilcoxon signed ranks test. Bonferroni's adjustments for multiple tests were used to confirm the validity of significant results. Relationships between changes in indices of apnoea severity and upper airway calibre and shape were studied by linear regression analysis.
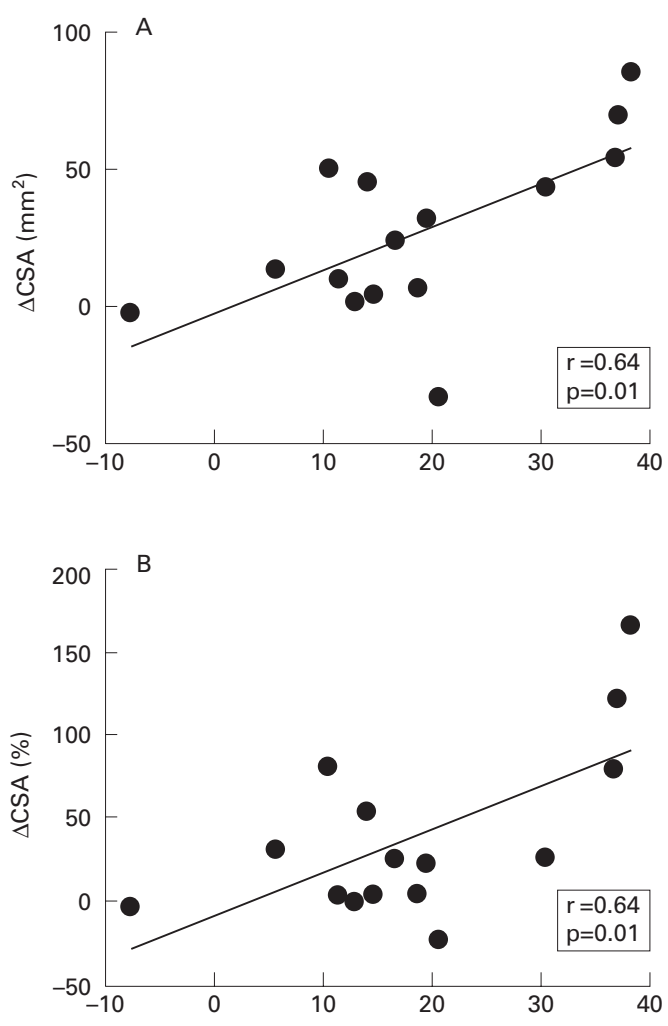

$\Delta \mathrm{AHI}$

Figure 4 Relationship between change in apnoea + hypopnoea index ( $\triangle A H I)$ and $(A)$ the absolute and $(B)$ the proportional changes in cross sectional area ( $\triangle C S A)$ of the velopharynx after insertion of the Klearway ${ }^{T M}$ oral appliance.

\section{Results}

Anthropometric and polysomnographic data for the 15 patients are summarised in table 1 . The patients were middle aged (mean age 52 years (range 25-70)), moderately obese (BMI $32 \mathrm{~kg} / \mathrm{m}^{2}$ (range 23-65)), and had mild to moderate obstructive sleep apnoea (AHI 28/h (range 9-45)). The mean interval between the initial and follow up polysomnograms was four months (range 2-12). BMI did not change significantly over time. AI decreased from 4 $(0-38) / \mathrm{h}$ to $0(0-6) / \mathrm{h}(\mathrm{p}<0.01)$ and AHI from $28(9-45) / \mathrm{h}$ to $8(1-28) / \mathrm{h}(\mathrm{p}<0.001)$ following insertion of the oral appliance. All except one of the patients had a reduction in the severity of obstructive sleep apnoea associated with use of the oral appliance.

The videoendoscopic data are presented in table 2. Following insertion of the oral appliance the CSA of the upper airway increased by $18 \%$ (95\% CI 3 to 35 ), p<0.02, in the hypopharynx and by $25 \%$ (95\% CI 11 to 69 ), p<0.005, in the velopharynx (fig 2), but not significantly in the oropharynx. With the exception of the velopharynx, there were similar increases in the anteroposterior and lateral diameters of the upper airway resulting in little change in upper airway shape following oral appliance emplacement. In the velopharynx the increase in the lateral diameter was $14 \%$ (95\% CI 8 to 33$), \mathrm{p}<0.005$, indicating a predominant effect of the oral appliance on the lateral velopharyngeal walls (fig 3).

Using linear regression analysis we examined the relationship between the change in AHI 
and the absolute and proportional change in CSA of the upper airway. The decrease in AHI following insertion of the oral appliance was related to the increase in CSA in the velopharynx $(r=0.64 ; \mathrm{p}=0.01$; fig 4$)$. There was no relationship between the reduction in obstructive sleep apnoea severity and change in oropharyngeal or hypopharyngeal CSA.

\section{Discussion}

Our results indicate that the emplacement of a mandibular advancement oral appliance increases upper airway calibre during wakefulness in patients with mild to moderate obstructive sleep apnoea. The increase in CSA of the upper airway occurs predominantly in the velopharynx and, to a lesser extent, in the oropharynx and hypopharynx. In particular, the oral appliance increases the lateral diameter of the velopharynx. Furthermore, the reduction in severity of obstructive sleep apnoea associated with use of the oral appliance is related to the increase in awake calibre of the velopharynx. These findings suggest that the mandibular advancement oral appliance may reduce the severity of obstructive sleep apnoea by increasing the size of the velopharynx, particularly in its lateral dimension, thereby stabilising the upper airway and reducing its tendency to obstruct during sleep.

Although several studies have now documented the efficacy of mandibular advancement oral appliances in the treatment of obstructive sleep apnoea, ${ }^{12-14}$ few have examined the effects of these devices on upper airway dimensions. Schmidt-Nowara and colleagues $^{7}$ used lateral cephalometric radiographs to study 11 patients before and after emplacement of a dental orthosis and found that the posterior airway space increased by 3.2 (3.6) $\mathrm{mm}(\mathrm{p}<0.01)$. Bonham and associates ${ }^{5}$ studied 12 patients using lateral cephalometry and found an increase in the retropalatal, but not the retroglossal, airspace after emplacement of a modified functional appliance. Eveloff and coworkers ${ }^{8}$ studied 19 patients and found no change in the posterior airway space following insertion of an oral appliance. In a detailed study of a single patient using three dimensional computed tomographic reconstructions ${ }^{10}$ we have previously shown that a mandibular repositioning appliance reduced the AHI from $57 / \mathrm{h}$ to $2 / \mathrm{h}$, increased the posterior airway space from $9.4 \mathrm{~mm}$ to $10.2 \mathrm{~mm}$, and increased the minimum airway CSA from $41.6 \mathrm{~mm}^{2}$ to $92.3 \mathrm{~mm}^{2}$. None of these studies examined the relationship between the change in upper airway dimensions and the change in severity of obstructive sleep apnoea. Our results confirm the increase in upper airway dimensions associated with a mandibular repositioning appliance and extend these findings by showing a relationship between increased awake CSA of the upper airway and reduced severity of obstructive sleep apnoea.

Previous investigators have assumed that mandibular advancement oral appliances exert their effects predominantly in the oropharynx and hypopharynx, rather than the velopharynx. ${ }^{16}$ However, the relatively high success rates of these appliances in relieving obstructive sleep apnoea in patients unselected for the site of upper airway obstruction suggested an additional effect in the velopharynx, since this is the commonest site of upper airway obstruction during sleep in patients with obstructive sleep apnoea. While we did note that the oral appliance increased upper airway CSA in the hypopharynx and insignificantly in the oropharynx, a more important finding of our study was the observation that the oral appliance exerted its major and most consistent effect in the velopharynx. Furthermore, the success of this appliance in relieving obstructive sleep apnoea was related to its effect in increasing awake CSA in the velopharynx. We believe the efficacy of this oral appliance is related to its regional effects in the velopharynx. We hypothesise that the oral appliance, by displacing the mandible ventrally, places traction on the tongue and other ventral structures, which in turn tenses the anterior and posterior tonsillar pillars containing the palatoglossus and palatopharyngeus muscles, respectively. This action may displace the velum ventrally and alter the configuration of the velopharynx causing an increase in its lateral dimension also. We suspect that traction on the palatoglossus and palatopharyngeus muscles may increase the lateral diameter of the velopharynx and reduce its collapsibility, thus explaining the efficacy of the oral appliance in reducing the severity of obstructive sleep apnoea. The effect on the lateral dimension of the velopharynx is interesting since it has been shown that the velopharynx is more compliant laterally than anteroposteriorly. ${ }^{17}{ }^{18}$ Furthermore, different treatments that are effective in relieving obstructive sleep apnoea, such as nasal CPAP, uvulopalatopharyngoplasty (UPPP), and possibly weight reduction, may exert their effects predominantly on the lateral velopharyngeal walls. ${ }^{18} 19$

Neuromuscular factors are important determinants of upper airway patency during wakefulness and sleep. Loss of the neuromuscular compensatory mechanism that maintains upper airway patency during wakefulness is believed to be an important factor in upper airway obstruction during sleep in patients with obstructive sleep apnoea. ${ }^{20}$ Ventral displacement of the mandible, however, appears to improve upper airway patency regardless of the state of neuromuscular activation or whether the patient is awake or asleep. The chin lift and jaw thrust manoeuvres are well recognised techniques for establishing and maintaining upper airway patency during cardiopulmonary resuscitation and general anaesthesia. ${ }^{21}$ Isono and colleagues ${ }^{22}$ recently demonstrated that the jaw thrust manoeuvre performed in anaesthetised and paralysed patients with obstructive sleep apnoea increased the CSA of the velopharynx and oropharynx and shifted the pressure-area relationship of the pharynx in an upward direction, indicating decreased pharyngeal compliance. We recently showed that active mandibular protrusion in awake patients 
with obstructive sleep apnoea and normal subjects increased the CSA of the upper airway, particularly in the oropharynx and hypopharynx. ${ }^{11}$ The present study shows that an oral appliance that maintains the mandible in a protruded posture also increases the CSA of the upper airway. Although there are clear differences in the degree of neuromuscular activity of the upper airway muscles between these different experimental conditions, in each case the effect of ventral displacement of the mandible is to increase upper airway calibre. Because of the different experimental designs used in these studies it is not possible to compare the magnitude of change in CSA of the upper airway between them. The fact that ventral displacement of the mandible under such heterogeneous conditions increases the CSA of the upper airway suggests that mandibular advancement oral appliances maintain an increased CSA during sleep in patients with obstructive sleep apnoea. Any increase in the CSA of the upper airway will tend to tilt the balance of forces acting on the upper airway in favour of improved patency, and probably explains the efficacy of oral appliances in reducing the severity of obstructive sleep apnoea. That the effect of the Klearway $^{\mathrm{TM}}$ oral appliance was observed predominantly in the velopharynx is consistent with our current understanding that the retropalatal airway is the major site of upper airway obstruction in obstructive sleep apnoea.

Our study has some limitations. We examined the upper airway of awake patients whereas obstructive sleep apnoea occurs during sleep when the effect of neuromuscular factors on upper airway calibre is less. It is possible, though unlikely for the reasons outlined above, that sleep could eliminate the effects of oral appliance emplacement on upper airway patency. Preliminary data from Oshima et al indicate that a mandibular advancement oral appliance also increases the CSA of the upper airway and decreases upper airway compliance during sleep. ${ }^{23}$ These results will require confirmation in a larger patient sample. We studied patients after they had been using the Klearway $^{\mathrm{TM}}$ oral appliance for at least two months. It is possible that chronic use of the oral appliance might have induced some changes in upper airway configuration. Occasionally, patients who use a mandibular advancement oral appliance chronically have observed changes in their bite register in the morning which reverts to normal after several hours. These changes could be associated with a change in the CSA of the upper airway that persists after removal of the oral appliance. If so, the changes are likely to be small and in the direction of those found in this study. We have previously addressed the limitations of videoendoscopy as a technique for measuring the CSA of the upper airway. ${ }^{11}$

In summary, using videoendoscopy we have shown that a mandibular advancement oral appliance increases the CSA of the upper airway, particularly in the velopharynx. This effect on velopharyngeal CSA occurs mainly as a result of an increase in the lateral dimension of the airway. The efficacy of the oral appliance in reducing the severity of obstructive sleep apnoea is related to the increase in awake velopharyngeal size. This increase in velopharyngeal calibre during wakefulness may result in improved upper airway patency during sleep, possibly explaining the efficacy of oral appliance therapy in obstructive sleep apnoea.

The authors thank Mrs B Herbert for typing the manuscript This study was supported by the British Columbia Health Research Foundation and the Canadian Respiratory Health Network of Centres of Excellence (Inspiraplex).

1 Schmidt-Nowara W, Lowe A, Wiegand L, et al. Ora appliances for the treatment of snoring and obstructive appliances for the treatment of snoring and
sleep apnea: a review. Sleep 1995;18:501-10.

2 American Sleep Disorders Association. Practice parameters for the treatment of snoring and obstructive sleep apnea with oral appliances. Sleep 1995;18:511-3.

3 Clark GT, Arand D, Chung E, et al. Effect of anterior mandibular positioning on obstructive sleep apnea. Am Rev Respir Dis 1993;147:624-9.

4 Cartwright RD, Samelson CF. The effects of a nonsurgical treatment for obstructive sleep apnea. FAMA 1982;248 705-9.

5 Bonham P, Currier G, Orr WC, et al. The effect of a modified functional appliance on obstructive sleep apnea. $A m \mathcal{F}$ Orthod Dentofac Orthop 1988;94:384-92.

6 George P. A modified functional appliance for treatment of obstructive sleep apnea. 7 Clin Orthod 1987;21:171-5.

7 Schmidt-Nowara WW, Meade TE, Mays MB. Treatment of snoring and obstructive sleep apnea with a dental orthosis. Chest 1991:99:1378-85.

8 Eveloff SE, Rosenberg CL, Carlisle CC, et al. Efficacy of a Herbst mandibular advancement device in obstructive sleep apnea. Am Rev Respir Dis 1994;149:905-9.

9 Johnson LM, Arnett GW, Tamborello JA, et al. Airway changes in relationship to mandibular posturing. Otolaryngol Head Neck Surg 1992;106:143-8.

10 Lowe AA, Fleetham JA, Ryan CF, et al. Effects of a mandibular repositioning applicance used in the treatment of obstructive sleep apnea on tongue muscle activity. In: of Issa FG, Suratt PM, Remmers JE, eds. Sleep and respiration.

11 Ferguson KA, Love LL, Ryan CF. Effect of mandibular and tongue protrusion on upper airway size during wakefulness tongue protrusion on upper airway size during

12 Ferguson KA, Ono T, Lowe AA, et al. A randomized crossover study of an oral appliance vs nasal continuous positive airway pressure in the treatment of mild-moderate obstructive sleep apnea. Chest 1996;109:1269-75.

13 Clark GT, Blumenfeld I, Yoffe N, et al. A crossover study comparing the efficacy of continuous positive airway pressure with anterior mandibular positioning devices on patients with obstructive sleep apnea. Chest 1996;109: 1477-83.

14 Ferguson KA, Ono T, Lowe AA, et al. A short term controlled trial of an adjustable oral appliance for the treatment of mild to moderate obstructive sleep apnoea. Thorax 1997; 52:362-8.

15 Rechtschaffen A, Kales A, eds. A manual of standardized terminology, techniques and scoring systems for sleep stages of human subjects. UCLA, Los Angeles: Brain Information Service/Brain Research Institute, 1968

16 Bennett LS, Davies RJ, Stradling JR. Oral appliances for the management of snoring and obstructive sleep apnoea. Tho$\operatorname{rax}$ 1998;53(Suppl 2):58-64.

17 Kuna ST, Bedi DG, Ryckman C. Effect of nasal airway positive pressure on upper airway size and configuration. Am Rev Respir Dis 1988;138:969-75.

18 Schwab RJ, Pack AI, Guupta KB, et al. Upper airway and soft tissue structural changes induced by CPAP in normal subjects. Am 7 Respir Crit Care Med 1996;154:1106-16.

19 Welch KC, Goldberg AN, Trudo FJ, et al. Upper airway anatomic changes with magnetic resonance imaging in anatomic changes with magnetic resonance imaging in Med 1997;155:A938.

20 Mezzanotte WS, Tangel DJ, White DP. Waking genioglossus EMG in sleep apnea patients versus normal controls (a neuromuscular compensatory mechanism). 7 Clin Invest 1992;89:1571-9.

21 American Heart Association. Standards and guidelines for cardiopulmonary resuscitation. FAMA 1986;255:2905.

22 Isono S, Tanaka A, Sho Y, et al. Advancement of the mandible improves velopharyngeal airway patency. 7 Appl Physiol 1995;79:2132-8.

23 Oshima T, Vazquez JC, Masuda A, et al. Effects of incremental mandibular protrusion on cross-sectional area of the passive pharynx during medicated sleep in patients of the passive pharynx during medicated sleep in patients
with obstructive sleep apnea. Am $\mathcal{F}$ Respir Crit Care Med 1997;155:A676. 